\title{
Representing the intercultural development continuum as a pendulum: addressing the lived experiences of intercultural competence development and maintenance
}

\author{
Kris Acheson* \\ Center for Intercultural Learning, \\ Mentorship, Assessment and Research, \\ Purdue University, \\ Young Hall, Suite 120, 155 South Grant Street, \\ West Lafayette, IN, 47907, USA \\ Email: kacheson@purdue.edu \\ *Corresponding author
}

\section{Sundae Schneider-Bean}

Sundae Schneider-Bean LLC, Birkenweg 26, 3293 Dotzigen, Switzerland

Email: sundae@sundaebean.com

\begin{abstract}
While contemporary theories and models of intercultural competence (ICC) development such as the developmental model of intercultural sensitivity and the intercultural development continuum offer much insight for individuals and organisations that value effective, appropriate and satisfactory intercultural interactions, practitioners need a way to visualise these models that better aligns with the lived experiences of expatriates and other 'culture-crossers'. Representing the orientations of the intercultural development continuum on the arc of a pendulum rather than on a linear continuum not only more fully illustrates the fluid and complex nature of developing and maintaining intercultural competence but also highlights how competency has both globally applicable and locally dependent characteristics. In this article, a narrative of expat life is presented alongside a pedagogical tool that depicts stage-based intercultural competence development as a pendulum. Implications of using this metaphor to explicate stage-based development, including greater accessibility and reduced affective barriers, are discussed.
\end{abstract}

Keywords: cross-cultural; competency; intercultural development inventory; IDI; training; debriefing; adaptation; culture; orientation; DMIS.

Reference to this paper should be made as follows: Acheson, K. and Schneider-Bean, S. (2019) 'Representing the intercultural development continuum as a pendulum: addressing the lived experiences of intercultural competence development and maintenance', European J. Cross-Cultural Competence and Management, Vol. 5, No. 1, pp.42-61.

Biographical notes: Kris Acheson is the Associate Director of Intercultural Pedagogy and Scholarship at the Purdue University. She was raised in a military family with early exposure to world languages and cultures. She is a former faculty member in Applied Linguistics from the Georgia State 


\begin{abstract}
University, in 2010-2016. She earned an Interdisciplinary PhD in Intercultural Communication at the Arizona State University in 2008, after which her professional passion became mentoring students and colleagues towards greater intercultural competence (and training others to mentor). In 2015, she served as a US Fulbright Scholar at the Zamorano University in Honduras. She is an award-winning instructor, international education curriculum designer, and researcher with over two decades of classroom teaching experience and numerous publications and grants related to intercultural communication.

Sundae Schneider-Bean is an executive coach, experienced trainer and intercultural specialist. She is the Founder of Sundae Schneider-Bean LLC, Trailblazing Spouse ${ }^{\circledR}$, and the Expat Happy Hour (\#1 in iTunes in places and travel). She earned an MA in Intercultural Communication from the Arizona State University in 2006 and has lived and worked abroad for nearly 20 years. Drawing on her professional training, life experience and business background, she has coached clients from over 60 countries across six continents. She challenges her clients to reflect on their daily practices in order to develop intercultural competencies and communication skills.
\end{abstract}

This paper is a revised and expanded version of a paper entitled 'Representing the intercultural development continuum as a pendulum' presented at SIETAR-USA Annual Conference, San Diego, CA, USA, 18-21 October 2017.

\title{
1 Introduction
}

Many organisations and institutions, struggling with complex identity and communication issues in an increasingly globalised world, are investing in diagnosing and improving the intercultural competence (ICC), sometimes also referred to as 'emotional intelligence' (Early and Ang, 2003), of their leaders and members (Irving, 2010; Deardorff, 2006). The intercultural development inventory (IDI) is used frequently in both corporate and academic realms to measure ICC. The IDI is commonly used for two purposes: as a diagnostic tool, that is, to 'place' students or training participants into developmental stages in order to tailor curriculum that is stage-specific, and as an instrument of program evaluation administered in a pre/post intervention study design. However, the paradigm of ICC assessment has begun to shift in recent years towards a more learner-focused approach structured around formative assessment. Several quantitative instruments used for diagnostic and program evaluative purposes - for instance the intercultural effectiveness scale (http://www.kozaigroup.com) and the beliefs, events and values inventory (http:/www.thebevi.com) - emphasise their potential use for personal development. Like other measures, the IDI (http:// www.idiinventory.com) now offers participants a stage-specific development plan along with a report of results.

The IDI and its related theoretical frameworks, i.e., the developmental model of intercultural sensitivity (DMIS) (Bennett, 1986, 1993, 2004) and the intercultural development continuum (IDC) (Hammer, 2012, 2009) offer much insight for individuals and organisations that value effective, appropriate and satisfactory intercultural interactions. Some qualified administrators (QAs) of the IDI have found, however, that debriefing people on their IDI results can be problematic; they often encounter resistance 
to unexpectedly low scores and struggle to explain 'regression' over time because the model only projects forward growth. With IDI participants who find themselves in the midst of a challenge to their identity and/or worldview, it can be counterproductive to explain the process of development in a way that further unsettles their sense of self. Practitioners thus need a means of discussing ICC with those they mentor that better meets their developmental needs - a metaphor that articulates in a non-judgmental manner how and why people score on the IDI as they do. This article presents such a metaphor, representing the orientations of the IDC on the arc of a pendulum rather than on a linear continuum. Rather than an attack on the DMIS or IDC, the discussion below should be read as an effort to make these models both more accessible and more applicable to lived experiences. The implications of this innovation are discussed below, interwoven with a phenomenological narrative of one culture-crosser (the second author) whose story illustrates the fluid and complex nature of the development and the maintenance of ICC.

Sundae's story: "I grew up rather sheltered from other cultures in Williston, North Dakota, USA. I left for a study abroad semester in Toledo, Spain when I was twenty-one. Until that point, my cultural experiences had been comprised of fairly limited and superficial encounters with domestic diversity in the Mid-western USA and a few quick trips north to our 'neighbors' in Canada. In my early years I had no idea how important culture was to me, to others, or to my relationships with others, but in Spain I discovered how exciting it was to gain a glimpse of the world from another perspective. After finishing my degree in Spain, I added a few months exploring Europe and a whirlwind backpacking adventure in south-east Asia to my suite of experiences. In Vietnam, I met someone special and this led to a move to Bern, Switzerland at the age of twenty-two to commit to a relatively new relationship. I was starry-eyed, in love and hungry for cultural experiences. I soaked in the rich architecture of this UNESCO World Heritage Site; I tested out new foods and awkwardly learned how to properly greet friends. Bernese German (Bärnduutsch) presented a challenge, especially since I had to learn German (Hochdeutsch) to eventually understand it, but became well worth the effort. Even as I became more proficient in the language, though, I often found myself being taken off guard when I observed how the Swiss did certain things, or when I realized that they expected me to behave in ways that didn't feel natural to me. Sometimes the identity challenge, physical distance from family and sorrow for all I had given up brought me to my knees in tears. I tried to cope by focusing on all the things I had in common with the Bernese, the values we seemed to share and what good people they were.

Over the years I began to understand and even appreciate 'the way they do things here', including the importance of planning in advance, how friendships take more time to build and even the high value placed on security. I learned to tone it down in a restaurant or on public transport, being careful not to laugh too loudly and draw attention. Eventually I was able to integrate quite deeply, spending much of my time with Swiss friends and even heading up intercultural management at a major Swiss corporation. I was proud of the fact that I could train and coach my clients in German. I still felt a little different, but accepted. Over the years, Switzerland became 'home', so much so that I felt like an outsider when I went back to the USA to visit."

For those familiar with the W-curve and other models of cultural adaptation (Gullahorn and Gullahorn, 1963), as well as stage-based models of ICC development such as the DMIS (Bennett, 1993) and the IDC (Hammer, 2009), Sundae's story resonates as an illustration of intercultural growth and change. We can see her progress through stages of 
adaptation in the W-curve: honeymoon ('starry-eyed' and excited by difference), crisis ('awkward' and 'in tears'), adjustment ('understand' cultural values, 'learn to' behave in culturally appropriate ways and 'integrate deeply') and re-entry ('outsider' back in the US) phases. Likewise, we see her move through various intercultural orientations of the DMIS and the IDC in this narrative: denial ("no idea how important culture was"), polarisation (puzzlement over the expectations of the Swiss and romanticisation of all things foreign as 'exciting' and 'rich'), minimisation ('in common' and 'share'), acceptance ('understand and appreciate' new belief system and norms), and adaptation ('learned to' and 'could do'). However, Sundae's story does not end here. This is, in fact, only the beginning of her lifelong developmental process of learning to interact across cultures.

Contemporary theories and models of adaptation and ICC development offer much insight for individuals and organisations that value effective, appropriate and satisfactory intercultural interactions and thus strive to make progress towards more ethnorelative/intercultural ways of experiencing difference. Yet, because the models illustrated above are typically depicted with linear, unidirectional visual aids, they do not always fully resonate with the lived experiences of expatriates and other culture-crossers, as we will see when Sundae's narrative continues to unfold in the remainder of this article. As a collaboration between a practicing scholar and a scholarly practitioner who are well-steeped both in the academic traditions of ICC development and assessment and in the day-to-day praxis of mentoring others through these processes, this article weaves together Sundae's story with a new heuristic that presents the IDC as a pendulum rather than a linear continuum. This metaphor better represents the fluid and complex nature of not only the development of intercultural competency but also its maintenance across various shifting contexts and conditions. Furthermore, the depiction of the IDC orientations on a pendulum offers an explanation for how global and local aspects of competence interact with each other and manifest in individual experience. Following a review of relevant theoretical frameworks, we detail the applications of the pendulum metaphor, relate and analyse more of Sundae's story, and discuss important implications of this new pedagogical tool.

\section{Review of literature}

\subsection{Global versus local competencies}

Scholars and practitioners in the field of intercultural communication have long argued over the nature of ICC. The primary debate is the basic definition of the term. Although no discernible consensus has been reached on the components that contribute to ICC, there are several themes common to most descriptions, including the inclusion of various skills, knowledge and attitudes (Spitzberg and Changnon, 2009). One aspect of this debate most relevant to Sundae's story is the question of whether ICC is locally or globally applicable. In other words, having developed ICC in the Swiss context, will Sundae always fully carry that competency with her as she journeys into other unfamiliar cultural territories? Scholars who argue that ICC is global provide evidence of the transferability of skills and attitudes, while scholars who conceive of ICC as local point to the specificity of linguistic and cultural knowledge that does not necessarily transfer to other localities. Organisations must therefore define ICC, at least implicitly, before 
attending to its development, since different understandings of the construct suggest different courses of action in strategic planning (for instance, workshops in emotional resilience or immersive language training).

While most, at least in theory, acknowledge the global and local natures of ICC as a both/and dialectic rather than an either/or dichotomy, in practice, interculturalists tend to be rather pragmatic in their treatment of ICC, favouring either the local or global aspects of ICC based on which best serve their particular needs. For example, many intercultural trainers and scholars in corporate (Callen, 2008; Early and Mosakowski, 2004; Irving, 2010; Marcel, 2011) and military (Abbe et al., 2007; Gallus et al., 2014; Watson, 2010; Wolfel, 2008) contexts focus on more broadly applicable or globally transferable skills because they add value to the concept of ICC in these contexts. In these realms, institutions frequently measure the ICC (a.k.a. cultural intelligence, intercultural effectiveness and global competence) of individuals with an eye towards predicting successful outcomes at the organisational level. This type of measurement often operationalises ICC as relatively acontextual, atemporal and static once developed. In other words, organisations operating in this paradigm tend to assume that, once achieved by an individual, ICC is forever after present, regardless of contextual factors such as a move to another unfamiliar cultural setting or the physical and emotional resources available to that individual in any given moment. If, for instance, an employee has scored highly on an intercultural assessment, that person may then be judged ready for any and all intercultural challenges. This conclusion is especially problematic when self-report measures with high face-validity are used to determine intercultural competency, since this characteristic of an instrument often leads to the overestimation of skill level (see Brenner and DeLamater, 2016, for an explanation based on identity theory of how the actual, ought, and ideal selves can influence survey response). Moreover, individuals themselves contribute to the discourse emphasising the global nature of ICC when, armed with the evidence of their ICC assessment results, they assume the mantle of general cultural intelligence in order to increase their status or appeal during hiring, promotion, compensation, task assignment and other organisational processes.

Likewise for pragmatic reasons, many in the field of language acquisition (Byram et al., 2001; Genc and Bada, 2005; Witte, 2014) emphasise the local aspects of ICC over the global ones. Because their job security and the status of their field is in part dependent on evidence of the importance of what they teach, it seems natural that they would tend to highlight how vital linguistic skills and culture-specific knowledge are in successfully negotiating intercultural interactions. Discipline and organisational type are of course not the only influences on this debate. Dervin (2010) points out that paradigm can impact one's stance in the local/global debate, with postmodernists and poststructuralists pushing for more fluid, contextual and multi-layered understandings of ICC.

\subsection{DMIS and IDC}

Bennett's (1986) DMIS, foundational in the field, remains a highly influential theory for both scholars and practitioners (trainers, coaches, etc.) of ICC (Witte, 2014). The DMIS posits six stages on a developmental spectrum, ranging from the more ethnocentric orientations of denial, defence and minimisation to the more ethnorelative orientations of acceptance, adaptation and integration (Bennett, 1993). In the 1990s, an instrument called the IDI was created to assess intercultural sensitivity, placing individuals and groups who take the inventory on the spectrum of the DMIS (Hammer, 2009). Over years of validity 
and reliability testing including over 10,000 participants, much of it conducted by external researchers (e.g., Paige et al., 2003), the IDI has been refined into the current widely-used online version (V3) of 50 items scored on a 5-point Likert scale and a variety of customisable demographic and open-ended questions. Researchers have utilised the IDI in a variety of contexts and settings, for example with high school students in an international school (Straffon, 2003), US K12 teachers in a professional development program (DeJaeghere and Cao, 2009; DeJaeghere and Zhang, 2008), short and long-term study abroad students (Jackson, 2010; Vande Berg et al., 2009) and future physicians participating in an intercultural intervention (Altshuler et al., 2003). More recently, Bennett (2014) shifted the discourse about the DMIS from more positivist stages of development to more constructivist positions on a continuum, emphasising that "one position is predominant, although perceptual strategies may span several positions" (n.p.).

Grounded in years of data from the IDI, Hammer (2012) also recently presented a model, the IDC that differs somewhat from the DMIS and more closely aligns with the Inventory. Hammer argues that validity testing of the IDI supports a model (see Figure 1) with five stages instead of six, and he labels the ends of the spectrum with the terms monocultural mindset and intercultural mindset rather than ethnocentric and ethnorelative.

Figure 1 Intercultural development continuum (see online version for colours)

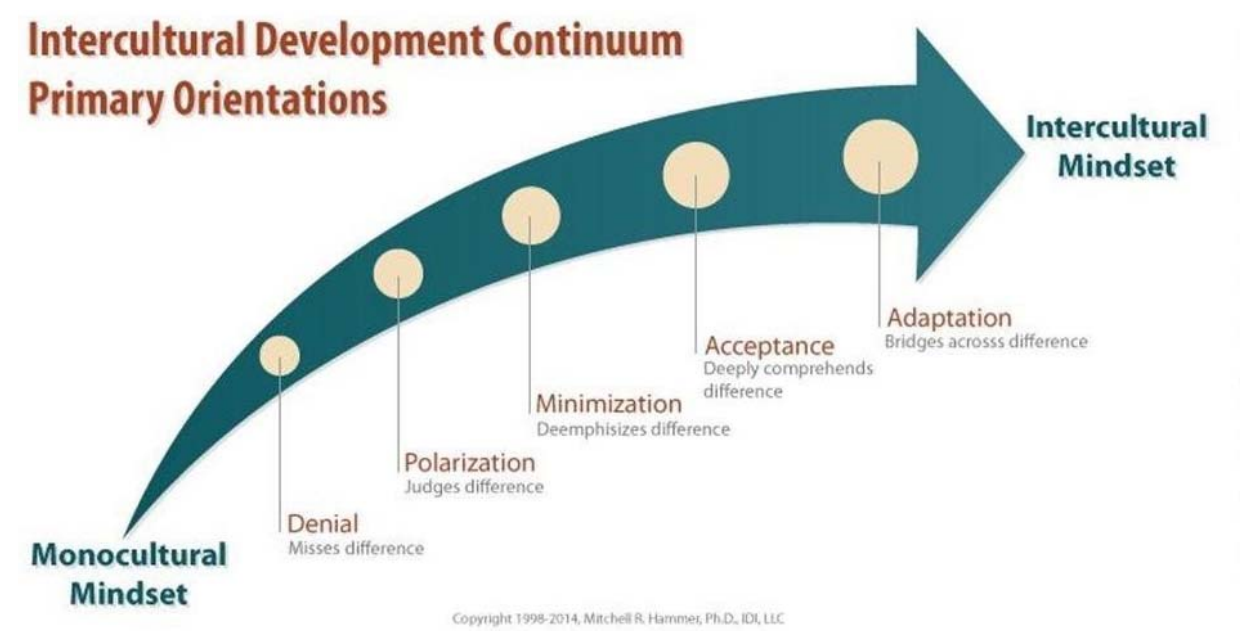

Source: IDI, LLC (2017)

Participants who take the IDI receive a report of results in individual and/or group debriefings with trained QAs of the instrument. The report places the inventory-taker(s) in one of these five orientations on the IDC on two separate scales, the first for one's perceived (subjective, or self-diagnosed) orientation and the second for one's developmental (objective, or instrument-diagnosed) orientation:

- Denial: an orientation that likely recognises more observable cultural differences (e.g., food) but may not notice deeper cultural difference (e.g., conflict resolution styles) and may avoid or withdraw from cultural differences. 
- Polarisation: a judgmental orientation that views cultural differences in terms of 'us' and 'them'. This can take the form of:

a Defence: an uncritical view toward one's own cultural values and practices and an overly critical view toward other cultural values and practices.

b Reversal: an overly critical orientation toward one's own cultural values and practices and an uncritical view toward other cultural values and practices.

- Minimisation: an orientation that highlights cultural commonality and universal values and principles that may also mask deeper recognition and appreciation of cultural differences.

- Acceptance: an orientation that recognises and appreciates patterns of cultural difference and commonality in one's own and other cultures.

- Adaptation: an orientation that is capable of shifting cultural perspective and changing behaviour in culturally appropriate and authentic ways (IDI, LLC, 2012).

In addition, the profile describes some strengths and weaknesses of the orientation and discusses Trailing Orientations, or the unresolved earlier orientations that one might revert to in stressful moments.

The DMIS and IDC models have not escaped critique. Witte (2014) points out that the models are less about behavioural ICC and more about a person's subjective experience of difference, and also criticises the fit of the DMIS for students of foreign language since it fails to fully acknowledge the role of language in facilitating interactions and shaping identities. Other scholars have questioned the IDI's construct and ecological validity (Matsumoto and Hwang, 2013). Moreover, some assumptions of the models are not always borne out in empirical research.

For example, even with the nuances of IDC Trailing Orientations reported by the IDI and Bennett's (2012) acknowledgement of the messiness of one's position on the DMIS spectrum, both models imply a fairly stable trajectory of growth in which ground once gained on the continuum may be lost again only momentarily. Yet, Van Der Poel (2016) found that study abroad participants moved significantly in both directions on the IDI. Likewise, Krishnan et al. (2017) noted that across the studies they reviewed where the IDI was used as a measure before and after an intervention, "14-31\% of participants declined" on the IDC (p.19).

In addition, some recent qualitative research has demonstrated how variable and nonlinear the opinions and emotions that reflect orientations can be over the course of mere weeks, and sometimes even expressed within the same paragraph in journaling (Garrett-Rucks, 2014). Human perspectives, in a word, are messy - much messier than the ways we tend to discuss them based on these linear models. As demonstrated with the empirical evidence above, ICC can be both multi-faceted and unstable in the moment and variable in the long-term - that is, not always clearly in a trajectory of positive gain or growth towards ethnorelative/multicultural perspectives (see Havril, 2015 for a discussion of an intercultural learning spiral, for instance). Such messiness may seem contradictory to the construct of development, which some would argue by its very nature requires directionality. However, the findings of these scholarly studies suggest that we should be thinking of directionality as optimal rather than inevitable; in other words, even when an optimal direction of development is indicated in a model, it does not necessarily follow that moving in another direction, or not moving at all, is impossible or even 
abnormal. Clearly, bi-directional movement on the IDC is a common phenomenon in published IDI data and should not be ignored.

From the point of view of a practitioner - an instructor, trainer or coach who often explains these models and IDI results to others - the DMIS and the IDC can be problematic in ways not discussed above. The clear, linear stage-by-stage progression from one orientation to the next that is such a strength in terms of the three main purposes of the IDI (diagnosis of group orientation for pedagogical planning, measure of the effectiveness of an intervention and aid for individual development of intercultural sensitivity) at times becomes a double-edged sword. Because they depict movement on the continuum as relatively unidirectional, it can be difficult for culture-crossers to understand all of their lived experiences in terms of these models.

For example, two factors affecting intercultural orientation that the DMIS and IDC do not emphasise are the interactions between global and local aspects of intercultural competency and the impact of expat fatigue. With regard to the first of these, as practitioners we have witnessed individuals not just lapsing momentarily into trailing orientations but living long-term in previously outgrown stages such as polarisation or minimisation when they transition into unfamiliar cultural environments after having already internalised orientations of acceptance and/or adaptation in a different context. With the second, in moments of crisis and with a cumulative effect over the long-term, the lack of physical and emotional resources that are symptomatic of 'cultural fatigue' [Szanton, (1966), p.36] can hamper the intercultural sensitivity of those who had previously achieved ethnorelative/multicultural orientations.

Perhaps most importantly, IDI QAs who regularly debrief others on their individual results are quite cognisant of the fact that IDI scores are not always well-received. Shock and disappointment, even disbelief, are common (Hofner Saphiere, 2012). These emotional reactions to debriefings can be taken as evidence of the validity of the instrument's distinction between the more subjective perceived orientation and the more objective developmental orientation (which is typically lower than the perceived). However, the validity of IDI scores does not mitigate the challenge for IDI administrators of debriefees' negative affect when 'lower' than expected or desired IDI scores are revealed in a debriefing. Perhaps even more than the IDI scores themselves, the perceived criticism of the imagery (i.e., an upward pointing arrow) and language (e.g., denial, polarisation, minimisation) used in discourse about the inventory can be counterproductive to positive learning experiences when the IDI is used as a formative assessment. The lived experiences of practitioners strongly suggest that a different way of talking about the orientations of intercultural sensitivity would be useful.

Despite some criticism, the DMIS and IDC, as well as the IDI assessment, remain well-represented in contemporary literature and continue to gain momentum in both corporate and academic arenas, where the development of ICC is often an explicitly stated and well-funded strategic organisation goal. Far from advocating their discontinuance, in this paper we argue that these models could become even more resonant with human experience and useful for ICC developmental work if framed differently. It is important to note at this point that, although the DMIS predates the IDC by many years and is considered the foundational model of stage-based ICC development, the remainder of this article discusses the IDC almost exclusively. We acknowledge the important contributions made by the DMIS, but because IDI results are framed in terms of the IDC (see IDI, LLC, 2012), and since we anticipate this 
pedagogical innovation being utilised most frequently in the context of discussing IDI results, this seems appropriate.

\section{The pendulum metaphor for intercultural development and maintenance}

To enhance its resonance with lived experience and reduce the implied judgment inherent in the current model's monocultural 'end', the five orientations of the IDC, which are normally depicted in a linear model along an arrow moving from left to right, can instead be mapped onto a pendulum as illustrated in Figure 2.

Figure 2 IDC orientations on a pendulum (see online version for colours)

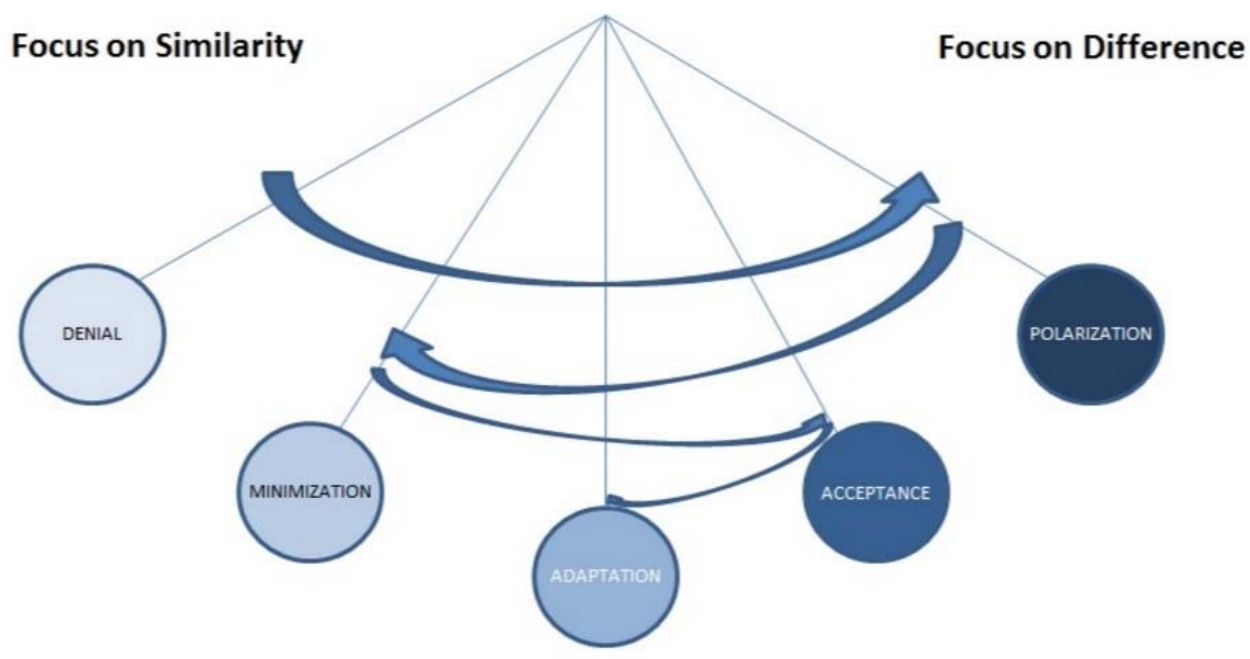

This pendulum swings between a focus on similarity and a focus on difference, with the orientations that have the most unbalanced focus on similarity (denial) and difference (polarisation) located at the most extreme reaches of the pendulum trajectory. Moving from one orientation to the next on the continuum (for example, from polarisation to minimisation) means swinging from one side to the other (in this case, from too much emphasis on difference to too much emphasis on similarity), with each swing less extreme until one may eventually find balance between the two in adaptation. Adaptation is situated midway between the two extremes because in this orientation, when we are able to successfully bridge cultural differences and behave appropriately in contexts beyond our native culture(s), we have resolved the dissonance between self and other, achieving a dialectic of similarity and difference.

Although human perspective is not subject to the laws of physics, the pendulum metaphor can be a powerful heuristic device for understanding lived experience. In Sundae's story above we see her start at the far left of this pendulum growing up with little exposure to difference, swinging to the far right with her fascination for experiences of the Other, moving partway back again towards similarity in her struggle to find connection in Switzerland, shifting somewhat towards difference once again as she learned to value Bernese perspectives. Finally she settled into a balance between 
similarity and difference when she could perform Swiss norms and speak German at a professional level, thus enabling her to embed herself deeply in her new community and work life while still feeling authentic in her interactions.

The pendulum metaphor does not fundamentally change the nature of stage-based development, which posits that each stage is a natural reaction to or outgrowth from the previous. More so than a straight line does, the metaphor explains the relationships between the stages by demonstrating why people move into polarisation from denial, into minimisation from polarisation, and so forth: like over-correcting a vehicle with sensitive steering, people swing too far towards the opposite of their current orientation before (hopefully) finding balance.

Figure 3 Unsettling the pendulum (see online version for colours)

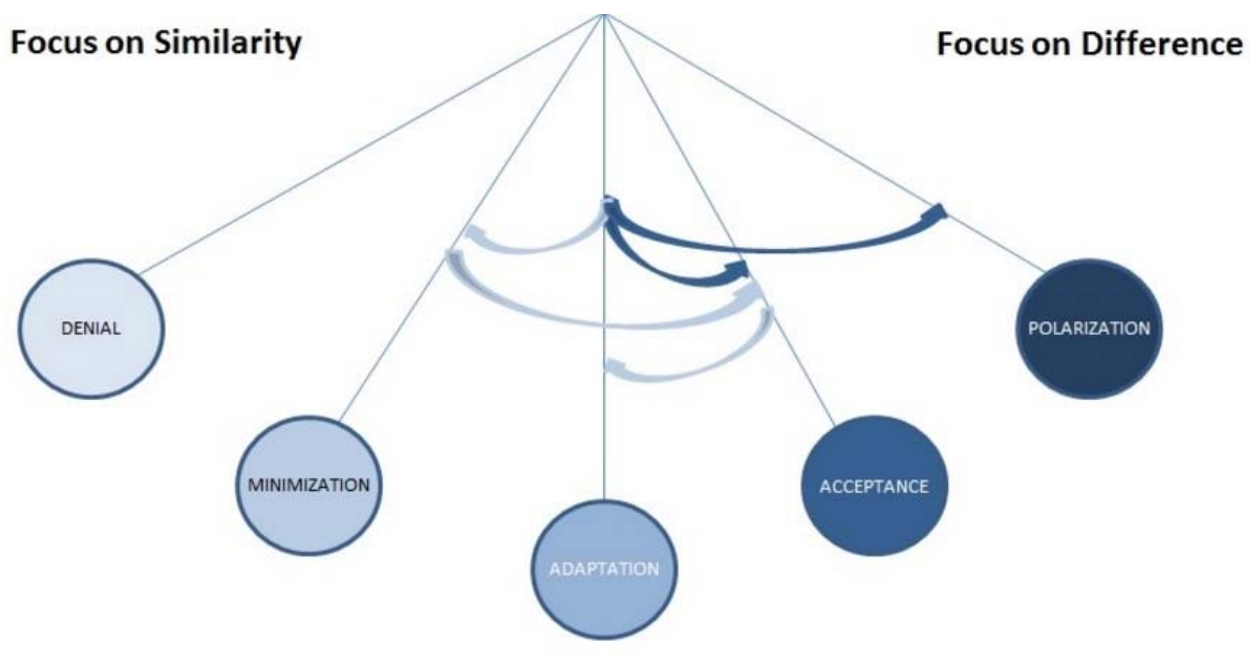

As illustrated in Figure 3, a pendulum, whether in motion or at rest, is disturbed by change. The IDC, pictured as an arrow pointing in one direction, implies that movement from stage to stage is unidirectional. While the trailing orientations reported in IDI profiles hint at variability, they do not fully capture either the long-term or short-term fluctuation that many people experience. In contrast, the pendulum metaphor acknowledges the possibility, even the likelihood, of motion in either direction (not only the optimal one). Even after having achieved adaptation, when we move to a new context our orientation becomes unsettled and begins to swing again back and forth between too much emphasis on similarity and on difference. This defensive reaction to a new environment is often a result of the loss of local knowledge and skills, so the more dramatic the change/the loss, the more extreme the swings of the pendulum are likely to be. However, many people entering new cultural waters after having already reached multicultural mindsets on the IDC do not swing all the way to the far extremes of the arc (e.g., all the way to denial) because they retain the global skills and attitudes they developed in their initial advancement through the stages. These same global skills and attitudes - for instance, openness, empathy, curiosity, skills of cultural discovery and mindfulness - can also help us return the pendulum to equilibrium more quickly and with less effort than would otherwise be possible. 
Sundae's story: "Three years ago, I did something many people thought was crazy. I left what I had worked so hard to build in Switzerland (the language, geographical familiarity, a strong network of friends, a rewarding and impactful job) to move to Ouagadougou, Burkina Faso (West Africa). I was no longer starry-eyed. I was an established professional and mother of two young children. I was leaving an immensely secure situation and diving into the unknown on so many levels. What had not changed is that I was still hungry for new cultural experiences. Moving to West Africa felt like going to a whole new world. On a reconnaissance trip to Ouagadougou my husband sent photos, reported on the infrastructure and gave his first impression of what the people were like. We agreed we were all in. We said goodbye to all we were leaving behind in Switzerland and landed in the airport on 5 August 2013.

I was responsible for training in the new nanny and helping the children adjust to the changes in the first few weeks. I did a lot of observing of how the Burkinabe greeted each other and how the men did not make as much direct eye contact as they do in Switzerland or in the USA. I wondered how I was ever going to learn how to drive in this seemingly perilous and erratic traffic. Every time I would go out in the city, I would just stare out the window of my car soaking in the views of the donkey carts, mothers carrying babies on their backs using only a simple piece of fabric and the live goat strapped to the top of a moving bus. What got loaded onto motorcycles seemed to be limitless: a family of four, full (raw) egg cartons stacked 20 high, live cows, or a large wooden door. It felt exotic. There were differences everywhere, and I was taking it all in. Some of the differences felt less playful when I experienced them, such as lower security measures at an amusement park resulting in an injury for my son, or waiting for the internet technician to come back... for six weeks instead of 'tomorrow at 9:30' as promised.

Another challenge is that I felt like a surgeon with oven mitts on when it came to speaking French. It was a deep plummet down from being able to interact professionally in fluent German to stumbling through my basic French and having Spanish resurface again when I had reached my French limits. I wanted to ask so many questions of the locals I was interacting with. I wanted to dive deep into discovering how things worked here, what was appropriate and how I should handle specific delicate situations. But, I simply didn't have the resources to express myself and dig out this gold mine of information."

Of course, not everyone moves through each stage to finally reach the equilibrium of adaptation, but no matter where one happens to have landed on the pendulum arc, change brings imbalance. Swings can be large-scale, moving you for a significant period of time to a previous orientation, or minor, shifting you from moment to moment between narrow-minded focus on similarity and difference. In Sundae's narrative above, we see her move overall from the orientation of adaptation in Switzerland to acceptance in her move to West Africa. Missing crucial local knowledge and communication skills ("I didn't have the resources to express myself"), she lacks the capacity to adapt as of yet, but still shows an appreciation for diversity ("the men did not make as much direct eye contact as they do in Switzerland") and a motivation to learn more ("I wanted to ask so many questions of the locals") that are typical of the acceptance orientation.

In addition to this variation in orientation that expats and other frequent culture-crossers experience over the long-term, fluctuation can also occur from day to day or even moment to moment. As the IDI trailing orientations suggest, people tend to have favoured coping mechanisms that we employ in moments of stress. These behaviours and attitudes are likely reflective of stages behind (or previous to) our primary 
orientation. While coping is not synonymous with developing or adapting, coping strategies for cultural discomfort and ambiguity are arguably more robust and effective in more multicultural orientations. Furthermore, the lack of capacity to cope can inhibit development because moving into and remaining in multicultural orientations requires great emotional resilience. Sundae's story highlights times when she caught herself swinging to polarisation-reversal by romanticising her new home ("What got loaded onto motorcycles seemed to be limitless... It felt exotic") and polarisation-defence when she made more negative comparisons ("lower security measures at an amusement park resulting in an injury for my son, or waiting for the internet technician to come back... for six weeks"). Clearly, even those who have moved into acceptance and adaptation orientations are susceptible to this short-term instability. However, the physics of the pendulum still apply: those starting from a more multicultural orientation (closer to the centre) will experience less extreme swings and can return to balance more quickly and easily. Those unsettled from minimisation or polarisation swing with more momentum since they begin farther from the centre. Consequently, they may find that recovering from moments of disequilibrium require more work and/or time.

The pendulum illustrates how and why these fluctuations in orientation occur, as well as how and why we can return fairly easily to our more typical orientations. Just as a physical pendulum responds sensitively to forces of nature such as gravity, propulsion, and inertia, this model is responsive to our lived realities, and the inevitable nature of the movement of the pendulum can bring relief from the judgment, shame and frustration that many feel in response to the linear and unidirectional IDC model. These short-term pendulum swings offer hope of more permanent movement along the continuum as well; they may even be the method by which we achieve the development of intercultural sensitivity, in that we proceed forward to more multicultural orientations in fits and starts, returning to our comfortable, current orientation often before fully making the transition to the next stage.

If change can be likened to propulsion, an external force that causes the pendulum to swing, we must also account for other forces that affect the pendulum's behaviour. Two of the most important of these are magnets and anchors. Magnets are exterior forces which tend to pull the pendulum towards one side or the other. Figure 4 provides several examples of magnets that pull us towards a focus on either similarity or difference. For instance, a rhetoric of religion that emphasises the common humanity shared by self and other (i.e., "we are all God's children") encourages us to concentrate on similarity and ignore cultural differences, as does an expat lifestyle that isolates us from the local community, leading us to believe that life abroad is not all that different from life back home. Magnets that pull us towards a focus on difference include moments when we are confronted with strikingly unfamiliar environments, behaviours and belief systems, as well as dissatisfying encounters with others filled with uncertainty, conflict or unmet expectations. Tourist lifestyles are listed on both sides: A hotel-and-guided-tour type of cultural experience might buffer us from local customs and blind us to significant cultural differences (pulling towards similarity); on the other hand, the exoticisation of foreign cultures that undergirds much tourism - that is, the performance of local rituals as a spectacle for the enjoyment of visitors rather than for their original purpose and the commodification of cultural artefacts as souvenirs - can encourage us to frame the world as us vs. them (pulling towards difference). 
Figure 4 Forces that act on the pendulum (see online version for colours)

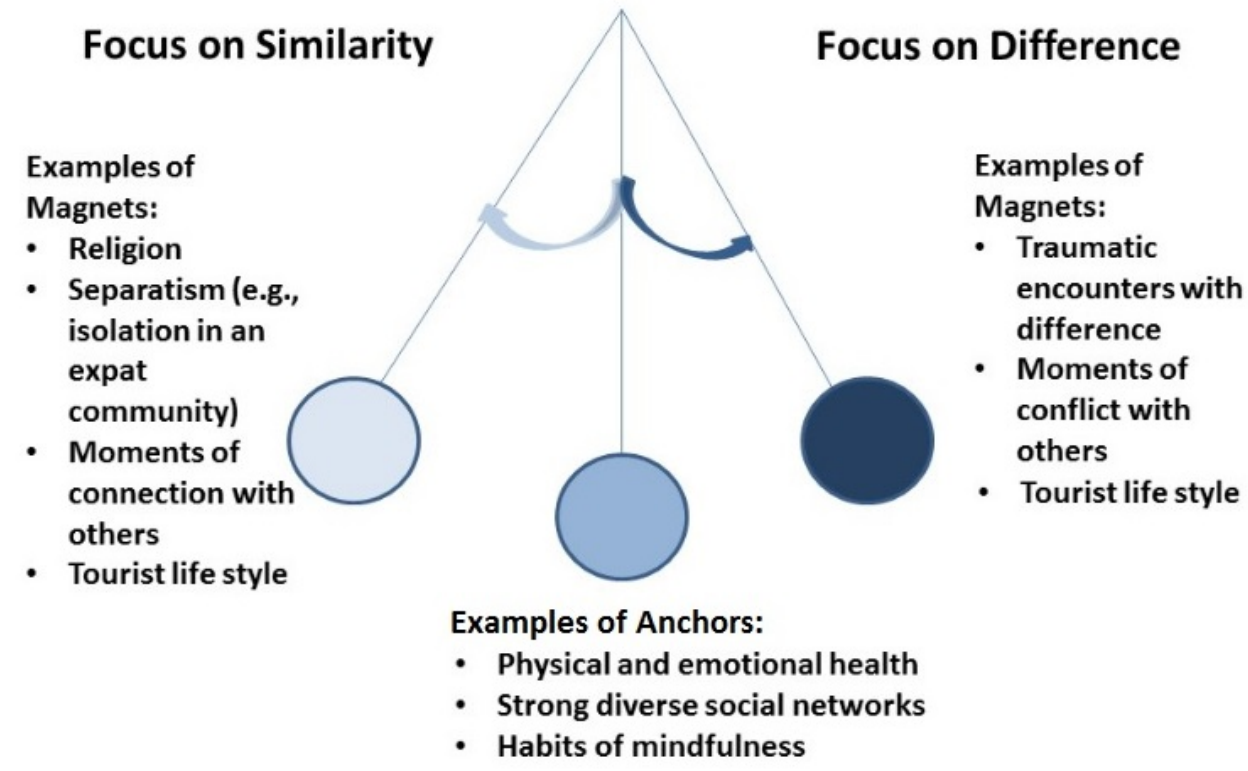

Like magnets, anchors also exert an attractive force on the pendulum, but rather than pulling you away from your habitual orientation, anchors ground you and allow you to resist swinging to previous stages on the IDC in times of stress. While magnets operate on the pendulum largely outside of our control, anchors require deliberate cultivation on our part; we must drop anchors if we want them to help hold us in place. As noted in Figure 4 below, one anchor that works to limit the influence of magnets on our orientation is physical and emotional health. Getting enough sleep, eating well and managing stress are key strategies for reducing the number and extent of pendulum swings in response to change, as well as crucial elements of a plan for regaining equilibrium quickly. Such strategies of self-care, or "the practice of activities that individuals initiate and perform on their own behalf in maintaining life, health, and wellbeing," are essential to maintaining resilience in the face of stress [Dodd, (2007), p.4]. In fact, taking 'care of yourself' is the first strategy recommended by the Foreign Service Institute (2016, p.31) for building personal resilience in unfamiliar settings. Strong social networks can also function as an anchor, helping to stabilise us when life sets our pendulum swinging, by meeting our needs for emotional support and providing us with information that helps us understand and adapt to another culture. This second function acting as a source of information - is the reason why Figure 4 specifies 'diverse' social networks as an anchor. When our social networks are monocultural they may not help, and could even hinder, our capacity to move beyond over-emphasising either similarity or difference.

Sundae's story: "One welcome respite from the immense task of getting settled in Ouagadougou was how quickly I was able to meet a lovely group of friends and acquaintances. I felt immediately welcomed into the International School community. All of the patience and effort I had expended to make new friends 
of my own in Bern got turned on its head in Burkina. Instead of my husband introducing me to his community, I was the one making connections within the expat community while my husband was at work. I was surrounded by families who were also raising Third Culture Kids. The downside to this fast-track community is that my needs for connection were being met by expats, thereby curbing the 'burn' to meet Burkinabe friends. There were also so many barriers, it seemed, to connecting meaningfully with the Burkinabe.

Another aspect that helped me in terms of community and making friends is that I had spent the last 15 years practicing how to have relationships with my loved ones from a distance. I knew that no matter where I was on the planet that I could stay connected, and this sense of security gave me some inner stability.

A year into our life in Africa, I really needed some stability. Two days after we returned from a vacation to Switzerland, the WHO declared a state of emergency in West Africa due to the Ebola outbreak. I had never been challenged quite on this level before. I felt so responsible as a parent with two small children to protect. I struggled to find a balance between being prudent and rational. There were times when I battled an urge to withdraw from the local community, 'just in case' they were in physical contact with a relative who was affected. I recall when the hype around Ebola was at a high that I was hesitant to touch the guard outside of the bank I frequented every week so instead began to greet him with "Bonjour! Ça va? Et la famille?" sans handshake.

A few months later, the panic around Ebola had subsided, but political uncertainty started to mount, with a declaration of civil disobedience by the opposition to President Blaise. This disobedience campaign led to the ousting of the President in a political uprising on 31 October 2014. The critical nature of these events and their inherent risks kept me focused on staying safe and functioning as normally as possible. These were not moments when I was pondering how I could expand my French vocabulary or develop relationships with locals.

Normal life quickly returned to Ouagadougou after the political uprising, but uncertainty remained with a transitional government and elections ahead. This uncertainty coupled with a level of heat that could melt any store of patience took its toll. In February 2015 I started writing about 'expat fatigue' for my blog because I was seeing hints of it all around me. Exaggerated responses to small disruptions, heightened frustrations with daily life and a general feeling of lethargy were just a few of the signs. I didn't want to let this fatigue hijack the joy that I had for the life and culture in Ouaga. But, I noticed I could respond to change so much more playfully when I had gone for a run or made time for a night out with my girlfriends. Likewise, on the evenings where I was able to slip away and read a bit or journal before heading to bed, I felt more energized in the mornings. These things grounded me. Whenever I got a bit sloppy about taking good care of myself, though, I felt more vulnerable and allowed myself to be more quickly frustrated by disruptions of my daily routine, such as power or water cuts. By being really diligent about prioritizing my health, my connections to others and time for myself, I could bring myself back to 'me'. Restoring my balance allowed me to better match the Burkinabe spirit of optimism, friendliness and demonstration of mutual respect. So when a local contact unexpectedly dropped by to say hello, I could see the visit not as an interruption, but rather as a pleasant surprise and a welcome opportunity to stop what I was doing, speak about the latest with the family and drink a bottle of cold soda." 
Sundae's continuous effort to maintain her ICC detailed in the narrative above clearly illustrates how magnets and anchors have impacted her adaptation to life in Burkina Faso. Her 'fast-track community' of expats, though an important source of support during her transition ('welcomed', 'surrounded'), pulled her towards similarity, preventing her from deeply experiencing her unfamiliar environment ("barriers to connecting meaningfully with the Burkinabe"). In contrast, the health and political crises pulled her towards difference, focusing her attention on the more stressful elements of her life ("battled an urge to withdraw", "hesitant to touch", and "moments when I was not pondering how I could expand my French vocabulary or develop relationships with locals"). Expat fatigue became another magnet for her, sapping her emotional resilience ("exaggerated responses to small disruptions, heightened frustrations with daily life, and a general feeling of lethargy") and capacity to adapt. Anchors also abound in her discussion, however, such as the conscientious maintenance of long-distance relationships with family and friends ("sense of security gave me some inner stability") and dedication to self-care ("I felt more energized in the mornings", "these things grounded me"). The pendulum metaphor thus provides a way to articulate not only the environmental forces acting upon Sundae in this context, as magnets would upon a pendulum, but also the strategies she can employ to counteract their impact on her, maintaining a healthy and balanced perspective ("not as an interruption, but as a pleasant surprise and a welcome opportunity") in the same way that an anchor offers stability.

\section{Implications}

The pendulum metaphor is a powerful tool for understanding the development and maintenance of ICC in part simply because we humans live, think and experience the world metaphorically, perceiving phenomena in terms of previously experienced and/or culturally constructed phenomena (Lakoff and Johnson, 1980). Metaphors work by abstracting characteristics of a SOURCE and applying them to a TARGET. For example, we might use a more familiar or more concrete object like money to understand a harder-to-grasp concept like time, mapping what we know of the SOURCE onto the TARGET. Thus, Kövecses (2002) argues that any model making extensive use of a metaphor to understand a phenomenon must necessarily highlight some aspects of an experience (where SOURCE and TARGET overlap) and obscure others (where they diverge). It is therefore important to explicitly acknowledge how metaphorical thinking both enables and limits understanding. This particular use of a pendulum metaphor to visually depict the stages of the IDC is highly beneficial because the metaphor:

- Emphasises the fluctuating nature of intercultural sensitivity: unlike a linear and unidirectional depiction of development, a pendulum encourages us to think about our orientation towards difference as more variable over time and across contexts, which resonates with the lived experience of many.

- Marks the relationship between global and local aspects of ICC: unlike primarily global or local characterisations of ICC that, respectively, overestimate or underestimate the transferability of one's competencies to new contexts, the pendulum offers a realistic sense of the partial/temporary shifts in effectiveness across difference that is likely to occur when we enter new cultural territory, carrying with us some global competencies and lacking some local ones. 
- Justifies stage-based development theory: with people who resist the idea of stage-based development (for example, questioning why one could not just move straight from polarisation to adaptation), the pendulum visually demonstrates the normalcy of swinging to the other side before finding balance in the centre.

- Illustrates some of the factors that de/stabilise one's orientation: thinking about how laws of physics may act upon a pendulum also allows us to articulate why our orientation shifts from stage to stage both in the long and short-term, and how we can attempt to counterbalance the effect of magnets (external forces/events that destabilise) with anchors (deliberate strategies that stabilise).

- Demonstrates the necessity of the purposeful maintenance of intercultural sensitivity after its initial development: this analysis of magnets and anchors discourages complacency in those who have reached more multicultural orientations, especially adaptation, and motivates us instead towards mindfulness, self-care and other strategies of maintaining hard-won progress on the IDC.

- Alleviates the shame and frustration of moving 'backwards' on the IDC and in fact of being in any particular orientation at any time: because a hierarchy of the stages on the IDC is not implied as much in a pendulum arc as in an arrow moving at an upwards diagonal, the pendulum presents stage-based development in a less judgmental way and allows for a different debriefing discourse, one comprised of phrases such as 'not as balanced' and 'closer to the centre' rather than 'low scores' and 'more advanced' orientations.

One drawback of the pendulum metaphor that must be acknowledged, however, is the dangerous implication that moving through the stages of the IDC to more multicultural orientations is a naturally-occurring and inevitable process, like the settling of a pendulum over time. In fact, because of the strong pull of the magnets of our experiences towards either side, moving towards the centre requires motivation, effort and resources. Moreover, staying centred demands all three, sustained over time and across contexts. The first of these requirements, motivation, is complex both because the types and intensity of motivation to interact successfully across difference varies from person to person and even with the same person in different situations, and because it is not only one's own attitudes that are important. Although some people, upon encountering cultural differences, experience motivation to adapt driven by a need for belonging and acceptance, Berry's (2005) decades of work on acculturation make it clear that interacting successfully across difference is much more complicated than one person's attitude and will; also highly impactful on outcomes are our treatment by others and the resources, information and accommodation that are made available to us by a host society.

Like motivation, effort and resources are necessary prerequisites of centring the pendulum and keeping it centred, and the pendulum metaphor fails to adequately emphasise these factors. A pendulum is deceptively inclined towards stillness; it wants to rest in balance. Humans are not often so effortlessly stationary. Consider the practice of meditation. Intense and sustained concentration is not typically an innate skill; instead, for most, a great deal of training, intentionality and practice are necessary to still our minds and bodies. Similarly to achieving a meditative state (although the pendulum metaphor does not highlight this aspect of our orientations towards difference), it is in 
fact quite difficult to reach equilibrium between similarity and difference at the stage of adaptation, and then to maintain that orientation over time. We have to earn the development of intercultural sensitivity, and as soon as we relax and stop being mindful of difference, or get distracted by compelling magnets that pull us towards either similarity or difference, or grow weary and no longer have the resources to commit to the work of staying balanced, we begin to swing again.

\section{Conclusions}

In short, while not without its metaphorical limitations, this pedagogical innovation of representing the IDC as a pendulum contributes much to our understanding of the development and maintenance of intercultural sensitivity, offering benefits both for individuals striving to understand their own intercultural adaptation processes and for trainers, educators and coaches attempting to design effective curriculum or programs to prepare and support people crossing cultures. It also bridges a disconnect between intercultural scholars and practitioners, in that it makes stage-based development theory more accessible to those not as familiar with academic literature or conversant on intercultural communication theories. Because the pendulum encourages us to expect swings, facilitates our recognition of swings when they happen, gives culture-crossers a language and structure to identify and productively address magnets and anchors and, perhaps most importantly, normalises movement in all directions on the IDC, the pendulum metaphor is a valuable addition to the discourse on ICC. To professionals preparing to live abroad as expatriates, it is a warning that their cultural intelligence may not carry over fully to their current or next context, as well as a reassurance about their capacity to recover. To students participating in study abroad programs, it is an explanation for their reactions to uncomfortable experiences and a suggestion of strategies for gaining and keeping balance. To instructors, advisors and coaches mentoring people through intercultural transitions, it is a reminder to offer continued support beyond pre-departure orientations or cultural training. To organisations, it is a heuristic for understanding the challenges, needs and developmental processes of members experiencing expatriation and repatriation or struggling to navigate a diverse workplace. For all of us, it is a means to deeper self-awareness and more realistic expectations of our own and others' ICC as well as a language with which to discuss interacting across difference that resonates more fully with our lived experience.

\section{Disclaimer}

This research did not receive any specific grant from funding agencies in the public, commercial or not-for-profit sectors. 


\section{References}

Abbe, A., Gulick, L.M.V. and Herman, J.L. (2007) Cross-cultural Competence in Army Leaders: A Conceptual and Empirical Foundation, October, United States Army Research Institute for the Behavioral and Social Sciences, Leader Development Research Unit, Arlington, VA.

Altshuler, L., Sussman, N.M. and Kachur, E. (2003) 'Assessing changes in intercultural sensitivity among physician trainees using the intercultural development inventory', International Journal of Intercultural Relations, Vol. 27, No. 4, pp.387-401.

Bennett, M. (2012) 'Turning cross-cultural contact into intercultural learning', in Proceedings of the Universidad 2012 8th International Congress on Higher Education, The University for Sustainable Development, Havana, Cuba.

Bennett, M.J. (1986) 'A developmental approach to training for intercultural sensitivity', International Journal of Intercultural Relations, Vol. 10, No. 2, pp.179-195.

Bennett, M.J. (1993) 'Towards ethnorelativism: a developmental model of intercultural sensitivity', in Paige, R.M. (Ed.): Education for the Intercultural Experience, 2nd ed., pp.21-71, Intercultural Press, Yarmouth, ME.

Bennett, M.J. (2004) 'Becoming interculturally competent', in Wurzel, J. (Ed.): Toward Multiculturalism: A Reader in Multicultural Education, 2nd ed., pp.62-77, Intercultural Resource Corporation, Newton, MA.

Berry, J.W. (2005) 'Acculturation: living successfully in two cultures', International Journal of Intercultural Relations, Vol. 29, No. 6, pp.697-712.

Brenner, P.S. and DeLamater, J. (2016) 'Lies, damned lies, and survey self-reports? Identity as a cause of measurement bias', Social Psychology Quarterly, Vol. 79, No. 4, pp.333-354.

Byram, M., Nichols, A. and Stevens, D. (Eds.) (2001) Developing Intercultural Competence in Practice, Multilingual Matters, Clevedon, UK.

Callen, D. (2008) 'How intercultural competence drives success in global virtual teams', Graziadio Business Review, Vol. 11, No. 4 [online] https://gbr.pepperdine.edu/2010/08/howintercultural-competence-drives-success-in-global-virtual-teams/ (accessed 31 May 2018).

Deardorff, D.K. (2006) 'Identification and assessment of intercultural competence as a student outcome of internationalization', Journal of Studies in International Education, Vol. 10, No. 3, pp.241-266.

DeJaeghere, J.G. and Cao, Y. (2009) 'Developing U.S. teachers' intercultural competence: does professional development matter?', International Journal of Intercultural Relations, Vol. 33, No. 5, pp.437-447.

DeJaeghere, J.G. and Zhang, Y. (2008) 'Development of intercultural competence among US American teachers: professional development factors that enhance competence', Intercultural Education, Vol. 19, No. 3, pp.255-268.

Dervin, F. (2010) 'Assessing intercultural competence in language learning and teaching: a critical review of current efforts', in Dervin, F. and Suomela-Salmi, E. (Eds.): New Approaches to Assessment in Higher Education, pp.157-173, Peter Lang, Bern.

Dodd, M. (2007) 'Intervening with self-care', International Cancer Nursing News, Vol. 19, No. 1, pp.4-5.

Early, P.C. and Ang, S. (2003) Cultural Intelligence: Individual Interactions Across Cultures, Stanford University Press, Stanford, CA.

Early, P.C. and Mosakowski, E. (2004) 'Cultural intelligence', Harvard Business Review, Vol. 82, No. 10, pp.139-46. 
Foreign Service Institute (2016) Foreign Service Assignment Notebook: What do I do Now?, United States Department of State, Washington, D.C., Transition Center and George P. Shultz National Foreign Affairs Training Center [online] https://www.state.gov/documents/ organization/258710.pdf (accessed 31 May 2018).

Gallus, J.A., Gouge, M.C., Antolie, E., Fosher, K., Jasparre, V. et al. (2014) Cross-cultural Competence in the Department of Defense: An Annotated Bibliography, April, United States Army Research Institute for the Behavioral and Social Sciences, Arlington, VA.

Garrett-Rucks, P. (2014) 'Measuring instructed language learners' IC development: discrepancies between assessment models by Byram and Bennett', International Journal of Intercultural Relations, Vol. 27, pp.467-486.

Genc, B. and Bada, E. (2005) 'Culture in language learning and teaching', The Reading Matrix, Vol. 5, No. 5, pp.73-84.

Gullahorn, J.T. and Gullahorn, J.E. (1963) 'An extension of the U-curve hypothesis', Journal of Social Issues, Vol. 14, No. 3, pp.33-47.

Hammer, M. (2012) 'The intercultural development inventory: a new frontier in assessment and development of intercultural competence', in Vande Berg, M., Paige, R.M. and Lou, K.H. (Eds.): Student Learning Abroad, pp.115-136, Stylus Publishing, Sterling, VA.

Hammer, M.R. (2009) 'The intercultural development inventory', in Moodian, M.A. (Ed.): Contemporary Leadership and Intercultural Competence, pp.203-218, Sage, Thousand Oaks, CA.

Havril, A.K. (2015) 'Improving intercultural competence of female university students in EFL within Saudi Arabia', Procedia: Social and Behavioral Sciences, Vol. 192, pp.554-566.

Hofner Saphiere, D. (2012) 'What do you mean?! I've worked abroad 20 years and score low?!', Cultural Detective Blog, 6 November [online] https://blog.culturaldetective.com/2012/11/06/ what-do-you-mean-ive-worked-abroad-20-years-and-score-low/.

IDI, LLC (2012) IDI Sample Individual Profile [online] https://idiinventory.com/wp-content/ themes/evolution/pdfs/Jose__Exemplar___Profile___August_2012.pdf (accessed 31 May 2018).

IDI, LLC (2017) The Intercultural Development Continuum (IDC $\left.{ }^{\mathrm{TM}}\right)$ [online] https://idiinventory.com/products/the-intercultural-development-continuum-idc/ (accessed 31 May 2018).

Irving, J.A. (2010) 'Educating global leaders: exploring intercultural competence in leadership education', Journal of International Business and Cultural Studies, Vol. 3, pp.1-14.

Jackson, J. (2010) 'Habitus, self-identity, and positioning: the multifarious nature of study abroad', Cultus: The Journal of Intercultural Mediation and Communication, Vol. 3, pp.65-78.

Kövecses, Z. (2002) Metaphor: A Practical Introduction, Oxford University Press, New York.

Krishnan, L.A., Masters, C., Holgate, H., Wang, C. and Calahan, C.A. (2017) 'Structured study abroad enhances intercultural competence', Teaching and Learning in Communication Sciences and Disorders, Vol. 1, No. 1, Article 5, pp.1-26.

Lakoff, G. and Johnson, M. (1980) Metaphors we Live By, University of Chicago Press, Chicago.

Marcel, M.F. (2011) 'Intercultural competence will save the day', in Meadows, A.J. and Tapia, A. (Eds.): Global Diversity Primer, Diversity Best Practices, New York.

Matsumoto, D. and Hwang, H.C. (2013) 'Assessing cross-cultural competence: a review of 17 available tests', Journal of Cross-Cultural Psychology, Vol. 44, No. 6, pp.849-873.

Paige, R.M., Jacobs-Cassuto, M., Yershova, Y.A. and DeJaeghere, J. (2003) 'Assessing intercultural sensitivity: an empirical analysis of the Hammer and Bennett intercultural development inventory', International Journal of Intercultural Relations, Vol. 27, Nos. 467-486, 4pp.

Spitzberg, B.H. and Changnon, G. (2009) 'Conceptualizing intercultural competence', in Deardorf, D. (Ed.): The Sage Handbook of Intercultural Competence, pp.1-52, Sage, Thousand Oaks, CA. 
Straffon, D.A. (2003) 'Assessing the intercultural sensitivity of high school students attending an international school', International Journal of Intercultural Relations, Vol. 27, No. 4, pp.487-501.

Szanton, D. (1966) 'Cultural confrontation in the Philippines', in Textor, R.B. (Ed.): Cultural Frontiers of the Peace Corps, pp.35-61, MIT Press, Cambridge, MA.

Van Der Poel, M.H. (2016) 'When does study abroad foster intercultural competence: a study in search of conditions', European Journal of Cross-Cultural Competence and Management, Vol. 4, No. 2, pp.168-185.

Vande Berg, M., Connor-Linton, J. and Paige, R.M. (2009) 'The Georgetown Consortium Project: interventions for student learning abroad', Frontiers: The Interdisciplinary Journal of Study Abroad, Vol. 18, pp.1-75.

Watson, J.R. (2010) 'Language and culture training: separate paths?', Military Review, No. 2, pp.93-97.

Witte, A. (2014) Blending Spaces: Mediating and Assessing Intercultural Competence in the L2 Classroom, Walter de Gruyter, Boston.

Wolfel, R.L. (2008) Culture Cubed: Towards Three-part Definition of Intercultural Competence, Center for Languages, Culture and Regional Studies, US Military Academy [online] http://www.usma.edu/clcrs/siteassets/sitepages/publications/clcrs\%20position\%20paperwhat $\% 20$ is\%20culture.pdf (accessed 31 May 2018). 